\title{
First-principles prediction of structure, energetics, formation enthalpy, elastic constants, polarization, and piezoelectric constants of AIN, GaN, and InN: Comparison of local and gradient-corrected density-functional theory
}

\author{
Agostino Zoroddu, Fabio Bernardini, and Paolo Ruggerone \\ INFM and Dipartimento di Fisica, Università di Cagliari, Italy \\ Vincenzo Fiorentini \\ INFM and Dipartimento di Fisica, Università di Cagliari, Italy \\ and Walter Schottky Institut, Technische Universität München, Garching, Germany
}

(Received 24 November 2000; published 2 July 2001)

\begin{abstract}
A number of diverse bulk properties of the zinc-blende and wurtzite III-V nitrides AlN, GaN, and InN, are predicted from first principles within density-functional theory using the plane-wave ultrasoft pseudopotential method, within both the local density approximation (LDA) and generalized gradient approximation (GGA) to the exchange-correlation functional. Besides structure and cohesion, we study formation enthalpies (a key ingredient in predicting defect solubilities and surface stability), spontaneous polarizations and piezoelectric constants (central parameters for nanostructure modeling), and elastic constants. Our study bears out the relative merits of the two density-functional approaches in describing diverse properties of the III-V nitrides (and of the parent species $\mathrm{N}_{2}, \mathrm{Al}, \mathrm{Ga}$, and In). None of the two schemes gives entirely successful results. However, the GGA associated with the multiprojector ultrasoft pseudopotential method slightly outperforms the LDA overall as to lattice parameters, cohesive energies, and formation enthalpies of wurtzite nitrides. This is relevant to the study of properties such as polarization, vibrational frequencies, elastic constants, nonstochiometric substitution, and absorption. A major exception is the formation enthalpy of $\mathrm{InN}$, which is underestimated by the GGA $(\sim 0$ vs $-0.2 \mathrm{eV})$.
\end{abstract}

DOI: $10.1103 /$ PhysRevB.64.045208

PACS number(s): 71.15.Mb, 61.50.Ah, 61.50.Lt

\section{INTRODUCTION AND METHOD}

The III-V nitride semiconductors AlN, GaN, and InN and their alloys are by now well established as a strategic material system ${ }^{1}$ for applications in high-frequency optoelectronics (light-emitting diodes and lasers), and high-power electronics (e.g., high-electron-mobility transistors). Most of their potential in these fields is due, respectively, to the large tunability of band gaps with alloy composition (in principle, 1.9 to $6.2 \mathrm{eV}$ ), and to their high peak and saturation drift velocity, coupled with polarization-induced effects allowing for the realization of high-density low-dimensional charge gases. $^{2}$

Nitride physics posed a number of puzzles to (and profited considerably from) $a b$ initio studies of various properties and subsystems, ranging from surfaces ${ }^{3}$ to defects, ${ }^{4}$ and polarization-related properties. ${ }^{5}$ Heralding the unusual nature of these materials, the standard study of the structural properties of bulk materials gave unexpected results in early studies. For instance, since some of the earliest papers, ${ }^{6}$ quite unusually for III-V semiconductors, the semicore $3 d$ electrons of Ga were found to behave as valence electrons and to be essential to describe accurately the structural properties.

A major source of uncertainty, both technical and ideological in nature, in density-functional theory (DFT) calculations is the choice of the exchange-correlation functional. While the local density approximation (LDA) is used most commonly, the generalized gradient approximation (GGA) has become a close competitor in recent years. In this work, we study the effects of using either LDA or GGA in the prediction of the properties of III-V nitrides. Such a comparison has been attempted only once previously for III-V nitrides, ${ }^{7}$ and restricted to structural and cohesive properties. $^{8}$

In this paper, we add a number of aspects to the theme of GGA versus LDA comparison for the nitrides. First, we use ultrasoft pseudopotentials, which should in principle $^{9}$ improve over norm-conserving potentials. ${ }^{7}$ Second, we calculate formation enthalpies, which are a cornerstone for predictions on nonstoichiometric systems relevant to surface reconstruction and impurity solubility. This calculation requires the study of the metallic phases of $\mathrm{Al}, \mathrm{Ga}$, and $\mathrm{In}$, the $\mathrm{N}_{2}$ molecule, and solid nitrogen (a molecular solid comprising $\mathrm{N}_{2}$ dimers on an hep lattice). Third, we evaluate the spontaneous polarization and the piezoelectric constants of the wurtzite phase ${ }^{10}$ in both the GGA and LDA. We find that these quantities are moderately affected by the choice of exchange correlation, unlike most others properties. Fourth, we evaluate a subset of the elastic constants in LDA and GGA.

The calculations have been done using VASP (Vienna $a b$ initio simulation package), ${ }^{11}$ which implements the DFT scheme within both the LDA and GGA approximations: we adopted the well established Perdew-Wang (PW91) version of the $\mathrm{GGA}^{12}$ and the Ceperley-Alder LDA. ${ }^{13}$ Ultrasoft pseudopotentials ${ }^{9}$ describe the electron-ion interaction. As usual, the potentials provided with VASP are generated for the free atom using the appropriate (LDA or GGA) functional. The pseudopotentials for $\mathrm{Ga}$ and In include, respectively, the semicore $3 d$ and $4 d$ states in the valence. A plane wave basis is used to expand the wave functions. We use a 
TABLE I. Bond length, vibrational frequency, and binding energy (not including zero-point energy) of the $\mathrm{N}_{2}$ dimer.

\begin{tabular}{llcc}
\hline \hline & $d(\AA)$ & $\omega(\mathrm{THz})$ & $E_{b}(\mathrm{eV})$ \\
\hline LDA & 1.107 & 464.3 & -11.332 \\
GGA & 1.113 & 442.8 & -10.558 \\
Experiment $^{\mathrm{a}}$ & 1.10 & 444.8 & -9.9 \\
\hline \hline
\end{tabular}

${ }^{\text {a Reference } 7 .}$.

cutoff of $350 \mathrm{eV}$, which is sufficient to fully converge all properties of relevance. For $k$-space summation, we use at least a Monkhorst-Pack (888) grid, except for the $\mathrm{N}_{2}$ molecule. Lattice constants and internal parameters are calculated using standard total energy calculations. Polarizations and related quantities are obtained using the Berry-phase approach $^{14}$ as in previous work. ${ }^{10}$ The elastic constants are calculated numerically as derivatives of the stress tensor, the stress values being taken at strains of about $\pm 1 \%$ along the $c$ and $a$ axis, for $C_{33}$ and $C_{31}$, respectively. Cohesive energies are calculated relative to spin-polarized free atoms. The formation enthalpies $\Delta H_{X \mathrm{~N}}$ per atom pair of the $X \mathrm{~N}$ crystals are calculated as

$$
\Delta H_{X \mathrm{~N}}=E_{X \mathrm{~N}}-E_{X}-E_{\mathrm{N}}
$$

where $E_{X \mathrm{~N}}$ is the total energy per atom pair of the compound $X \mathrm{~N}, E_{X}$ the energy per atom of bulk $X=\mathrm{Al}, \mathrm{Ga}$, and In, and $E_{\mathrm{N}}$ is the energy per $\mathrm{N}$ atom in the $\mathrm{N}_{2}$ dimer or the condensed $\mathrm{N}_{2}$ phase. We compare our results for the structure of the nitrides mostly with the LDA and GGA calculations by Stampfl and van de Walle. ${ }^{7}$ Many more theoretical data on structure are collected in Ref. 7.

\section{PARENT SPECIES}

\section{A. Nitrogen: Molecule and solid}

The nitrogen dimer is studied in artificial periodic conditions in a cubic box of side $10 \AA$, using the $\Gamma$ point for $k$ summation. The results, listed in Table I, agree well with other LDA and GGA calculations. GGA shows an overall better agreement with experiment. The binding energy is evaluated including the spin-polarization energy of the $\mathrm{N}$ atom $(-2.89 \mathrm{eV})$, calculated with a local-spin-density allelectron scalar-relativistic atomic code. ${ }^{15}$ Here, and below, we neglect the difference of spin-polarization energy in GGA and LDA. We checked that this causes an error per atom in the cohesive energy, of at most $10 \mathrm{meV}$ (40 meV for $\mathrm{N}_{2}$ ).

TABLE II. Structural parameters and binding energy per molecule (not including zero-point energy) of hexagonal solid $\mathrm{N}_{2}$.

\begin{tabular}{llll}
\hline \hline & $a(\AA)$ & $c / a$ & $E_{b}(\mathrm{eV})$ \\
\hline LDA (present) & 4.0205 & 1.3311 & -11.660 \\
GGA (present) & 4.0633 & 1.7929 & -10.701 \\
Experiment $^{\mathrm{a}}$ & 4.039 & 1.6514 & \\
\hline \hline
\end{tabular}

${ }^{\mathrm{a}}$ Reference 16 .
TABLE III. Lattice constant, binding energy, and bulk modulus of bulk fcc Al.

\begin{tabular}{lllc}
\hline \hline & $a(\AA)$ & $E_{b}(\mathrm{eV})$ & $B(\mathrm{Mbar})$ \\
\hline LDA (present) & 3.9809 & -4.064 & 0.766 \\
GGA (present) & 4.0491 & -3.561 & 0.689 \\
Experiment $^{\mathrm{a}}$ & 4.05 & -3.39 & 0.773 \\
\hline \hline
\end{tabular}

${ }^{\mathrm{a}}$ Reference 18.

Solid nitrogen is a condensate of $\mathrm{N}_{2}$ molecules. We consider the stable phase, with vertically oriented $\mathrm{N}_{2}$ molecules centered at the lattice points of a close packed hexagonal lattice. The (888) grid is used for $k$-space summation. We compare our results with experimental data from Ref. 16 in Table II. From the data in Tables I and II, the binding energy per molecule in the condensed phase is $0.328 \mathrm{eV}$ in the LDA and $0.143 \mathrm{eV}$ in the GGA. While performing well with respect to the in-plane lattice constant (about $\pm 0.5 \%$ relative deviation), both functionals fail to some extent with the axial lattice parameter: LDA underestimates it strongly $(\sim 20 \%)$ and GGA overestimates it $(\sim 9 \%)$. The vertical center-tocenter intermolecular distances are $3.33 \AA$ experimentally, $3.64 \AA$ in GGA, and $2.68 \AA$ in LDA. This system is indeed a severe test for both functionals because of its weak dipolar binding. GGA performs slightly better, as expected. The binding of the $\mathrm{N}_{2}$ system, already extremely large in reality, is overestimated appreciably in both approaches.

Our calculated zero-point energies for $\mathrm{N}_{2}$ are $0.153 \mathrm{eV}$ in the LDA, and 0.146 in the GGA. This reduces the binding of $\mathrm{N}_{2}$, and accordingly makes the formation enthalpies of the nitrides more negative by about $0.07 \mathrm{eV}$ in both cases. In the tables below, we will report the enthalpies without this additional energy. (We assume, as plausible, that the zero-point energy is the same for free and bound $\mathrm{N}_{2}$ molecules.)

\section{B. Bulk Al, Ga, and In}

Metallic Al, Ga, and In are a necessary ingredient to calculate formation enthalpies. Al is a good free-electron metal, and has fcc structure. Ga is a mixed-bonding marginal metal (see, e.g., Ref. 17). At ambient conditions, its stable phase is a dimerized structure known as $\alpha-\mathrm{Ga}$, a face-centered orthorhombic lattice with crystallographic vectors $\mathbf{a}_{1}=a \hat{\mathbf{x}}, \mathbf{a}_{2}$ $=\frac{1}{2}(b \hat{\mathbf{y}}+c \hat{\mathbf{z}}), \mathbf{a}_{3}=\frac{1}{2}(-b \hat{\mathbf{y}}+c \hat{\mathbf{z}})$ and eight atoms per primitive cell, whose positions are defined by two additional internal parameters $u$ and $v .^{16,17}$ Indium crystallizes in the

TABLE IV. Lattice constant, binding energy, axial ratios, and internal parameter (units of $c$ ) of $\alpha$-Ga.

\begin{tabular}{llllccc}
\hline \hline & $a(\AA)$ & $b / a$ & $c / a$ & $u$ & $v$ & $E_{b}(\mathrm{eV})$ \\
\hline LDA (present) & 4.4365 & 0.9985 & 1.6856 & 0.0816 & 0.1577 & -3.484 \\
LDA $^{\mathrm{a}}$ & 4.377 & 0.994 & 1.688 & 0.0803 & 0.1567 & \\
GGA (present) $^{4}$ & 4.5962 & 0.9917 & 1.6961 & 0.0834 & 0.1559 & -2.796 \\
Experiment $^{\mathrm{a}}$ & 4.51 & 1.0013 & 1.695 & 0.0785 & 0.1525 & \\
\hline
\end{tabular}

${ }^{\mathrm{a}}$ Reference 17. 
TABLE V. Lattice constant, axial ratio, and binding energy of bulk In.

\begin{tabular}{llll}
\hline \hline & $a(\AA)$ & $c / a$ & $E_{b}(\mathrm{eV})$ \\
\hline LDA (present) & 3.1861 & 1.5348 & -3.116 \\
GGA (present) & 3.2958 & 1.5448 & -2.470 \\
Experiment $^{\mathrm{a}}$ & 3.244 & 1.5222 & \\
\hline \hline
\end{tabular}

${ }^{a}$ Reference 16 .

monatomic body-centered tetragonal lattice, with lattice constants $a$ and $c$, and primitive vectors $\mathbf{a}_{1}=a \hat{\mathbf{x}}, \mathbf{a}_{2}=a \hat{\mathbf{y}}, \mathbf{a}_{3}$ $=\frac{1}{2}(a \hat{\mathbf{x}}+a \hat{\mathbf{y}}+c \hat{\mathbf{z}})$.

We report our results for $\mathrm{Al}$ in Table III, for Ga in Table IV, and for In in Table V. In these calculations we use a cutoff of $350 \mathrm{eV}$, an (888) $k$-space mesh for Al and In, and a (12 12 12) mesh for Ga. The cohesive energy includes the atomic spin-polarization energy $(-0.136 \mathrm{eV}$ for $\mathrm{Al}$, $-0.134 \mathrm{eV}$ for $\mathrm{Ga}$, and $-0.117 \mathrm{eV}$ for $\mathrm{In})$. The GGA improves appreciably the lattice constant and binding energy of Al. For Ga, both approaches are off by about the same amount in opposite directions for $a$. In both cases, axial ratios and internal parameters are excellent. Our LDA results are improved somewhat over those of Ref. 17, presumably because of the explicit treatment of $3 d$ electrons. For In, LDA and GGA are again off the mark by equal and opposite amounts for $a$. The LDA axial ratio is slightly better than that from the GGA. In short, the usual trend is obtained for the expanded and softer lattice as produced by GGA compared to LDA. If one is forced to choose, GGA generally performs better, especially in terms of cohesive energies. In any case, the deviations typically are below $\pm 1 \%$, so both approaches are quite legitimate.

\section{THE NITRIDES}

Binary III-V nitrides occur in nature in the wurtzite structure. Zinc-blende nitrides have a slightly higher energy. It is possible to grow epitaxially, e.g., zinc-blende $\mathrm{GaN}$ on cubic substrates. We first analyze zinc-blende (Sec. III A), then wurtzite (Sec. III B). Our results are compared with those of Ref. 7, where numerous other theoretical values are provided.

TABLE VI. Lattice constant, binding energy, and formation enthalpy $\Delta H$ of zinc-blende AlN.

\begin{tabular}{llll}
\hline \hline & $a(\AA)$ & $E_{b}(\mathrm{eV})$ & $\Delta H(\mathrm{eV})$ \\
\hline LDA (present) & 4.332 & -13.347 & -3.449 \\
LDA $^{\mathrm{a}}$ & 4.310 & -13.242 & \\
GGA (present) $_{\text {GGA }}^{\mathrm{a}}$ & 4.390 & -11.907 & -2.975 \\
Experiment $^{\mathrm{b}}$ & 4.394 & -11.361 & \\
\hline \hline
\end{tabular}

${ }^{\mathrm{a}}$ Reference 7.

${ }^{\mathrm{b}}$ Reference 7 .
TABLE VII. Lattice constant, binding energy, and formation enthalpy $\Delta H$ of zinc-blende GaN.

\begin{tabular}{lccc}
\hline \hline & $a(\AA)$ & $E_{b}(\mathrm{eV})$ & $\Delta H(\mathrm{eV})$ \\
\hline LDA (present) & 4.446 & -10.982 & -1.689 \\
LDA $^{\mathrm{a}}$ & 4.518 & -10.179 & \\
LDA $^{\mathrm{b}}$ & 4.466 & -10.880 & \\
GGA (present) $_{\text {GGA }^{\mathrm{a}}}^{4.538}$ & -9.249 & -1.102 \\
Experiment $^{\mathrm{c}}$ & 4.590 & -8.253 & \\
\hline \hline
\end{tabular}

${ }^{\text {a Reference } 7 .}$.

${ }^{\mathrm{b}}$ Reference 6.

${ }^{\mathrm{c}}$ Reference 7 .

\section{A. Zinc-blende AIN, GaN, InN}

For zinc-blende nitrides we used the usual $350 \mathrm{eV}$ cutoff and (888) $k$ grid. To estimate the cohesive energy, we use the atomic spin polarizations indicated previously. Our results are reported in Tables VI, VII, and VIII, for AIN, GaN, and InN, respectively. The results confirm the by now usual behavior of GGA versus LDA, consisting of a softening of the lattice, which improves lattice constant and binding energy, and worsens slightly the bulk modulus. Comparing the cohesive energy with that of the wurtzite phase as discussed below, we find that zinc blende is disfavored over wurtzite.

\section{B. Wurtzite AIN, Ga1N, InN}

Wurtzite is a hexagonal close-packed lattice, comprising vertically oriented $X-\mathrm{N}$ units at the lattice sites. The basal lattice parameter is $a$, the axial lattice parameter is $c$. The interatomic distance in the basic unit is described by an internal parameter $u$ expressed in units of the axial ratio $c / a$. The ideal (i.e., for touching hard spheres) values of the axial ratio and internal parameter are, respectively, $c / a=\sqrt{8 / 3}$ and $u=3 / 8$. The crystallographic vectors of wurtzite are a $=a(1 / 2, \sqrt{3} / 2,0), \mathbf{b}=a(1 / 2,-\sqrt{3} / 2,0)$, and $\mathbf{c}=a(0,0, c / a)$. The Cartesian coordinates of the basis atoms are $(0,0,0)$, $(0,0, u c), a(1 / 2, \sqrt{3} / 6, c / 2 a)$, and $a(1 / 2, \sqrt{3} / 6,[u+1 / 2] c / a)$.

Our results are reported in Tables IX, X, and XI, for AIN, $\mathrm{GaN}$, and $\mathrm{InN}$, respectively. For comparison, experimental data, and the results of Ref. 7 are also listed. As to structure, in all cases both the axial ratio and the internal parameter are nonideal. Deviation from ideality increases from $\mathrm{GaN}$ to $\mathrm{InN}$

TABLE VIII. Lattice constant, binding energy, and formation enthalpy $\Delta H$ of zinc-blende $\mathrm{InN}$.

\begin{tabular}{lllc}
\hline \hline & $a(\AA)$ & $E_{b}(\mathrm{eV})$ & $\Delta H(\mathrm{eV})$ \\
\hline LDA (present) & 4.964 & -9.232 & -0.282 \\
LDA $^{\mathrm{a}}$ & 5.004 & -8.676 & \\
GGA (present) $_{\text {GGA }^{\mathrm{a}}}$ & 5.067 & -7.680 & 0.140 \\
Experiment $^{\mathrm{b}}$ & 5.109 & -6.855 & \\
\hline \hline
\end{tabular}

${ }^{\mathrm{a}}$ Reference 7.

${ }^{\mathrm{b}}$ Reference 7 . 
TABLE IX. Lattice constant, axial ratio, internal parameter, and formation enthalpy of wurtzite AlN.

\begin{tabular}{lllccc}
\hline \hline & $a(\AA)$ & $c / a$ & $u$ & $E_{b}(\mathrm{eV})$ & $\Delta H(\mathrm{eV})$ \\
\hline LDA (present) & 3.0698 & 1.5995 & 0.3821 & -13.536 & -3.642 \\
LDA $^{\mathrm{a}}$ & 3.057 & 1.617 & 0.3802 & -13.286 & \\
$\mathrm{GGA}^{\text {(present })}$ & 3.1095 & 1.6060 & 0.3819 & -12.071 & -3.142 \\
GGA $^{\mathrm{a}}$ & 3.113 & 1.6193 & 0.3798 & -11.403 & \\
Experiment $^{\mathrm{b}}$ & 3.1106 & 1.6008 & $0.3821^{\mathrm{c}}$ & $-11.669^{\mathrm{a}}$ & $-3.13^{\mathrm{d}}$ \\
\hline \hline
\end{tabular}

${ }^{\mathrm{a}}$ Reference 7 .

${ }^{\mathrm{b}}$ Reference 20 .

${ }^{\mathrm{c}}$ Reference 21.

${ }^{\mathrm{d}}$ Reference 19 .

to AlN. As usual GGA improves considerably the binding energy, and occasionally the lattice constant, at the cost of a slight overestimate of the axial ratio. The internal parameter $u$ (alias the axial bond length) is well reproduced in all the various combination of materials and approximations. The experimental values of the lattice mismatch between the nitrides are well reproduced both by LDA and GGA. The maximum deviation from experimental mismatch is $0.3 \%$ for AlN/GaN, $1 \%$ for $\mathrm{GaN} / \mathrm{InN}$, and $1.3 \%$ for $\mathrm{AlN} / \mathrm{InN}$.

The GGA calculations produce lattice constants and internal parameters with maximum deviations from experiment below $0.3 \%$ for $\mathrm{AlN}, 0.9 \%$ for $\mathrm{GaN}$, and $1.7 \%$ for $\mathrm{InN}$ $(+1.3 \%$ for $a$ and $+1.7 \%$ for $c)$. In this respect, these are probably the best DFT pseudopotential results so far for these materials. The improvement over previous GGA results is to be attributed to the use of ultrasoft, multiprojector pseudopotentials. ${ }^{9}$ By the same token, it is quite likely that all-electron calculations using the same GGA parametrization may improve the agreement further, especially for $\mathrm{InN}$.

Calculated cohesive energies generally overestimate, as usual, the experimental value. GGA corrects in part the LDA overbinding, and exhibits better agreement. Comparing the cohesive energies of the zinc-blende and wurtzite phases, as already mentioned, we find wurtzite to be energetically favored over zinc blende. The predicted difference per atom pair between the two phases is $189 \mathrm{meV}$ (LDA) and 164 $\mathrm{meV}$ (GGA) for AlN, $17 \mathrm{meV}$ (LDA) and $16 \mathrm{meV}$ (GGA) for $\mathrm{GaN}$, and $17 \mathrm{meV}$ (LDA) and $15 \mathrm{meV}$ (GGA) for InN.

Good results are also achieved for the formation enthalpies. The values in the tables, referred to an atom pair, were

TABLE X. Lattice constant, axial ratio, internal parameter, and formation enthalpy of wurtzite $\mathrm{GaN}$.

\begin{tabular}{llllll}
\hline \hline & $a(\AA)$ & $c / a$ & \multicolumn{1}{c}{$u$} & $E(\mathrm{eV})$ & $\Delta H(\mathrm{eV})$ \\
\hline LDA (present) 3.131 & 1.6301 & 0.3768 & -10.999 & -1.685 \\
$\mathrm{LDA}^{\mathrm{a}}$ & 3.193 & 1.634 & 0.376 & -10.187 & \\
$\mathrm{GGA}^{(p r e s e n t)}$ & 3.1986 & 1.6339 & 0.3772 & -9.265 & -1.118 \\
$\mathrm{GGA}^{\mathrm{a}}$ & 3.245 & 1.632 & 0.3762 & -8.265 & \\
Experiment $^{\mathrm{b}}$ & 3.1890 & 1.6263 & 0.377 & $-9.058^{\mathrm{a}}$ & $-1.08^{\mathrm{c}}$ \\
\hline \hline
\end{tabular}

${ }^{\mathrm{a}}$ Reference 7.

${ }^{\mathrm{b}}$ Reference 21 .

${ }^{\mathrm{c}}$ Reference 19 .
TABLE XI. Lattice constant, axial ratio, internal parameter, and formation enthalpy of wurtzite InN.

\begin{tabular}{lllllr}
\hline \hline & $a(\AA)$ & $c / a$ & \multicolumn{1}{c}{$u$} & $E_{b}(\mathrm{eV})$ & $\Delta H(\mathrm{eV})$ \\
\hline LDA (present) & 3.509 & 1.6121 & 0.3791 & -9.249 & -0.303 \\
$\mathrm{LDA}^{\mathrm{a}}$ & 3.544 & 1.626 & 0.377 & -8.694 & \\
$\mathrm{GGA}^{\text {(present) }}$ & 3.5848 & 1.6180 & 0.37929 & -7.695 & 0.125 \\
$\mathrm{GGA}^{\mathrm{a}}$ & 3.614 & 1.628 & 0.377 & -6.872 & \\
Experiment $^{\mathrm{b}}$ & 3.538 & 1.6119 & & $-7.970^{\mathrm{c}}$ & $-0.21^{\mathrm{d}}$ \\
\hline \hline
\end{tabular}

${ }^{\mathrm{a}}$ Reference 7.

${ }^{\mathrm{b}}$ Reference 22.

${ }^{\mathrm{c}}$ Reference 7.

${ }^{\mathrm{d}}$ Reference 19.

obtained using the energy per $\mathrm{N}$ atom in the solid- $\mathrm{N}_{2}$ phase. If the $\mathrm{N}_{2}$ molecule is assumed as the reference instead, as is plausible in high-temperature growth techniques, the formation enthalpy becomes more negative by one-half of the binding energy of solid $\mathrm{N}_{2}$ - that is, $0.164 \mathrm{eV}$ and $0.071 \mathrm{eV}$, respectively, must be subtracted to the LDA and GGA values in the tables. To account for the zero-point motion of $\mathrm{N}_{2}$, half the zero-point energy of $\mathrm{N}_{2}(\simeq 0.07 \mathrm{eV})$ should be subtracted from the values in the tables.

The calculated GGA formation enthalpies are in general agreement with experiment for AlN and GaN. For InN, GGA overcorrects the LDA overbinding and gives a positive value. Using the free $\mathrm{N}_{2}$ molecule as the $\mathrm{N}$ reservoir, and including the zero-point energy, the GGA formation enthalpy of InN improves slightly, becoming essentially zero (in fact, barely negative but at the limit of our numerical accuracy). This problem is probably due to $\mathrm{InN}$ itself, and only marginally to the In or $\mathrm{N}$ parent phases. Indeed, quite unusually, even the calculated cohesive energy underestimates the experimental value. This was observed also in the pseudopotential study of Ref. 7 and in unpublished fullpotential linearized augmented plane wave calculations. ${ }^{24}$ We are not aware of other formation enthalpy calculations for InN. The issue is open to further investigation.

In Table XII, we report for each of the nitrides the spontaneous polarization in the equilibrium structure, the dynamical effective charges, the piezoelectric constants, and a subset of elastic constants relevant to symmetry-conserving strains. The reason for collecting these data in one table is that they provide an almost self-contained set of input data for the simulation of nanostructures made of wurtzite nitrides. The only additional data needed are the static dielectric constants, which were reported elsewhere. ${ }^{25}$ In the last column we report the proper piezoelectric constant $e_{31}^{\mathrm{p}}$. As discussed recently, ${ }^{26,27}$ this value should be compared with experiments involving current flow across the sample, whereas the "improper" constant $e_{31}$ is relevant to systems in depolarizing fields such as nitride nanostructures. ${ }^{5}$

It is not infrequent to hear the incorrect statement that the spontaneous polarization is nonvanishing in wurtzite because of structural nonideality. In actuality, a nonvanishing polarization is allowed on symmetry grounds ${ }^{28}$ in the ideal wurtzite structure as well. Indeed, we find that the calculated Berry-phase polarization in the ideal structure is 
TABLE XII. Spontaneous polarization $\left(\mathrm{C} / \mathrm{m}^{2}\right)$, piezoelectric constants $\left(\mathrm{C} / \mathrm{m}^{2}\right)$, dynamical charges, elastic constants $(\mathrm{GPa})$, and the ratio $R=-2 C_{31} / C_{33}$ (see text) of wurtzite nitrides, as obtained in the LDA and GGA approximation. The last column reports the proper $e_{31}$ piezoelectric constant.

\begin{tabular}{lccccccccc}
\hline \hline & $P$ & $Z^{*}$ & $e_{33}$ & $e_{31}$ & $C_{33}$ & $C_{31}$ & $R$ & $e_{31}^{\mathrm{p}}$ \\
\hline AlN & & & & & & & & \\
LDA & -0.100 & 2.652 & 1.80 & -0.64 & 384 & 111 & -0.578 & -0.74 \\
LDA $^{\mathrm{a}}$ & & & & & 373 & 108 & -0.579 & \\
GGA & -0.090 & 2.653 & 1.50 & -0.53 & 377 & 94 & -0.499 & -0.62 \\
GaN & & & & & & & & \\
LDA & -0.032 & 2.51 & 0.86 & -0.44 & 415 & 83 & -0.400 & -0.47 \\
LDA & & & & & 405 & 103 & -0.508 & \\
GGA & -0.034 & 2.67 & 0.67 & -0.34 & 354 & 68 & -0.384 & -0.37 \\
InN & & & & & & & & \\
LDA & -0.041 & 3.045 & 1.09 & -0.52 & 233 & 88 & -0.755 & -0.56 \\
LDA & & & & & 224 & 92 & -0.821 & \\
GGA & -0.042 & 3.105 & 0.81 & -0.41 & 205 & 70 & -0.683 & -0.45 \\
\hline \hline
\end{tabular}

${ }^{\mathrm{a}}$ Reference 23 .

$-0.032 \mathrm{C} / \mathrm{m}^{2}$ in $\mathrm{AlN},-0.018 \mathrm{C} / \mathrm{m}^{2}$ in $\mathrm{GaN}$, and $-0.017 \mathrm{C} / \mathrm{m}^{2}$ in $\mathrm{InN}$. These values are smaller (by a factor of 2 to 3) than the actual ones for nonideal structures (Table XII). This confirms the intuitive idea that nonideality, and especially changes in $u$, can increase polarization substantially, and indicates that an accurate determination of the structure is mandatory to obtain reliable polarization values. $^{27}$

Theoretical predictions on polarization properties were shown to compare quite favorably with experimental evidences in various papers (see, e.g., Refs. 2, 5, and 29). It should be noted, however, that the link between polarization and the observed quantities, typically optical shifts or densities of mobile charge, is rather indirect and affected by uncertainties due to issues of nanostructure design, material quality, and reverse modeling. Thus, comparison with experiment does not yet allow a clear-cut evaluation of the performance of LDA versus GGA. The recently discovered ${ }^{30}$ nonlinear behavior of the polarization in nitride alloys is an additional source of uncertainty.

The LDA elastic constants are in fair agreement with those of Wright. ${ }^{23}$ The GGA constants are smaller, as is to be expected given the general tendency of GGA to produce a softer lattice. According to elasticity theory, the axial strain induced in wurtzite by an in-plane (e.g., epitaxial) strain $\epsilon_{1}$ is $\epsilon_{3}=-2 \epsilon_{1} C_{31} / C_{33}=R \epsilon_{1}$. The quantity $R$ is thus relevant to epitaxial nitride systems, and it is reported in Table XII. Several experimental data for the elastic constants and $R$ are compiled in Ref. 23. The considerable spread of those data does not allow a definite conclusion about whether GGA produces a systematically improved agreement with experiment over LDA in this respect.

\section{SUMMARY}

In conclusion, the present calculations suggest an overall improvement of the predicted properties of wurtzite III-V nitrides through the GGA compared to the LDA. In particular, in the former approach, the structural parameters exhibit deviations from experiment below $0.3 \%$ for $\mathrm{AlN}, 0.9 \%$ for $\mathrm{GaN}$, and $1.7 \%$ for InN. GGA cohesive energies and formation enthalpies are in fair to excellent agreement with experiment, and improve over LDA values; the only clear-cut GGA failure is the formation enthalpy of InN. Elastic properties follow the expected trends of GGA versus LDA behavior; due to uncertainties in the experimental data, comparison with experiment does not provide definite support to one or the other approximation. Polarization properties are moderately sensitive to the exchange-correlation functional, as long as the latter predicts the correct structure (especially, the correct internal parameter $u$ ). For these properties too, comparison with experiment is indirect and affected by many sources of uncertainty, and does not support one or the other approach. Concerning the cohesion and structure of the parent species $\left(\mathrm{N}_{2}, \mathrm{Ga}, \mathrm{Al}, \mathrm{In}\right)$, only in the case of condensed $\mathrm{N}_{2}$ do we find major discrepancies with experiment.

In light of the present results, our conclusion is that the choice of either the GGA or LDA will depend on the specific problem being addressed. The GGA outperforms slightly the LDA overall with respect to cohesive energies and formation enthalpies of wurtzite nitrides (except for InN), and usually also as to lattice and internal parameters in comparison with recent accurate experiments. We thus presume that the GGA might be preferred in density-functional studies of III-V nitrides for quantities such as macroscopic polarization, piezoelectricity, lattice dynamics, and possibly elastic constants, which depend critically on the accuracy of the equilibrium structure.

\section{ACKNOWLEDGMENTS}

We thank Dr. A. Bosin for atomic calculations. Work at Cagliari University is supported in part by MURSTCofin99, and by INFM Parallel Computing Initiative. V. F. thanks the Alexander von Humboldt-Stiftung for support of his stay at the Walter Schottky Institut, and O. Ambacher for pointing out recent and accurate structural data.

\footnotetext{
${ }^{1}$ For a review see, e.g., O. Ambacher, J. Phys. D 31, 2653 (1988).

${ }^{2}$ O. Ambacher, J. Smart, J.R. Shealy, N.G. Weimann, K. Chu, M. Murphy, W.J. Schaff, L.F. Eastman, R. Dimitrov, L. Wittmer, M. Stutzmann, W. Rieger, and J. Hilsenbeck, J. Appl. Phys. 85, 3222 (1999).
}

\footnotetext{
${ }^{3}$ See, e.g., A.R. Smith, R.M. Feenstra, D.W. Greve, J. Neugebauer, and J.E. Northrup, Phys. Rev. Lett. 79, 3934 (1997).

${ }^{4}$ J. Neugebauer and C.G. van de Walle, Festkörperprobleme 35, 25 (1996).

${ }^{5}$ V. Fiorentini, F. Bernardini, F. Della Sala, A. Di Carlo, and P.
} 
Lugli, Phys. Rev. B 60, 8849 (1999).

${ }^{6}$ V. Fiorentini, M. Methfessel, and M. Scheffler, Phys. Rev. B 47, 13353 (1993); V. Fiorentini, A. Satta, D. Vanderbilt, S. Massidda, and F. Meloni, in The Physics of Semiconductors, edited by D. J. Lockwood (World Scientific, Singapore, 1995), p. 137.

${ }^{7}$ C. Stampfl and C.G. Van de Walle, Phys. Rev. B 59, 5521 (1999).

${ }^{8}$ Two other papers dealing with a GGA and LDA comparison, but with a more limited scope, have appeared recently. They dealt with the lattice constant and gap of cubic GaN in GGA [R. Miotto, G.P. Srivastava, and A.C. Ferraz, Phys. Rev. B 59, 3008 (1999)], and with the effects of GGA on transition pressures of GaN [M. Abu-Jafar, A.I. Al-Sharif, and A. Qteish, Solid State Commun. 116, 389 (2000)].

${ }^{9}$ D. Vanderbilt, Phys. Rev. B 41, 7892 (1990).

${ }^{10}$ F. Bernardini, V. Fiorentini, and D. Vanderbilt, Phys. Rev. B 56, R10 024 (1997).

${ }^{11}$ G. Kresse and J. Hafner, Phys. Rev. B 47, R558 (1993); G. Kresse, thesis, Technische Universität Wien, 1993; G. Kresse and J. Furthmüller, Comput. Mater. Sci. 6, 15 (1996); Phys. Rev. B 54, 11169 (1996).

${ }^{12}$ J. P. Perdew, in Electronic Structure of Solids '91, edited by P. Ziesche and H. Eschrig (Akademie-Verlag, Berlin, 1991), p. 11.

${ }^{13}$ D.M. Ceperley and B.J. Alder, Phys. Rev. Lett. 45, 566 (1980); J.P. Perdew and A. Zunger, Phys. Rev. B 23, 5048 (1981).

${ }^{14}$ R.D. King-Smith and D. Vanderbilt, Phys. Rev. B 47, 1651 (1993); D. Vanderbilt and R.D. King-Smith, ibid. 48, 4442 (1993); R. Resta, Rev. Mod. Phys. 66, 899 (1994).

${ }^{15}$ A. Bosin (unpublished).
${ }^{16}$ R. W. G. Wyckoff, Crystal Structures (Wiley, New York, 1963), Vol. I.

${ }^{17}$ M. Bernasconi, G.L. Chiarotti, and E. Tosatti, Phys. Rev. B 52, 9988 (1995).

${ }^{18}$ C. Kittel, Introduction to Solid State Physics, 6th ed. (Wiley, New York, 1986).

${ }^{19}$ D'Ans-Lax Taschenbuch für Chemiker und Physiker, Band I, edited by E. Lax and C. Synowietz (Springer, Berlin 1967).

${ }^{20}$ M. Tanaka, S. Nakahata, K. Sogabe, and H. Nakata, Jpn. J. Appl. Phys., Part 2 36, L1062 (1997).

${ }^{21}$ M. Leszczynski, H. Teisseyre, T. Suski, I. Grzegory, M. Bockowski, J. Jun, S. Porowski, K. Pakula, J.M. Baranowski, C.T. Foxon, and T.S. Chen, Appl. Phys. Lett. 69, 73 (1996).

${ }^{22}$ W. Paszkowicz, J. Adamczyk, S. Krukowski, M. Leszczynski, S. Porowski, J. A. Sokolowski, M. Michalec, and W. Lasocha, Philos. Mag. A 79, 1145 (1999).

${ }^{23}$ A.F. Wright, J. Appl. Phys. 82, 2833 (1997).

${ }^{24} \mathrm{M}$. Fuchs (private communication).

${ }^{25}$ F. Bernardini, V. Fiorentini, and D. Vanderbilt, Phys. Rev. Lett. 79, 3958 (1997).

${ }^{26}$ D. Vanderbilt, J. Phys. Chem. Solids 61, 147 (2000).

${ }^{27}$ F. Bernardini, V. Fiorentini, and D. Vanderbilt, Phys. Rev. B 63, 193201 (2001).

${ }^{28}$ G. Burns, Solid State Physics (Academic Press, New York 1985), pp. 88-92.

${ }^{29}$ P. Waltereit, O. Brandt, A. Trampert, H.T. Grahn, J. Menninger, M. Ramsteiner, M. Reiche, and K. Ploog, Nature (London) 406, 865 (2000).

${ }^{30}$ F. Bernardini and V. Fiorentini, Phys. Rev. B (to be published). 